\title{
Methodological Flaws in the Chabot Trial of Pancreatic Enzymes for Cancer Therapy
}

\author{
Colin A. Ross, M.D. \\ The Colin A. Ross Institute for Psychological Trauma \\ 1701 Gateway, \#349, Richardson, TX 75080, USA \\ rossinst@rossinst.com
}

\begin{abstract}
A trial of gemcitabine versus pancreatic enzymes for the treatment of pancreatic cancer reported an average survival time of 14 months for the chemotherapy group and 4.3 months for the enzyme group. Due to extensive missing information, readers of the paper reporting the trial would conclude that the study was valid. When this missing information is made available, as it is in the present report, it becomes evident that there were numerous serious methodological flaws in the study. No scientifically valid conclusions about the treatment of cancer with pancreatic enzymes can be reached from this study.
\end{abstract}

Keywords: pancreatic cancer, gemcitabine, pancreatic enzymes, clinical trial, methodological flaws.

\section{INTRODUCTION}

Chabot, Tsai, Fine, Chen, Kumah, Antman, and Grann [1] published a trial comparing gemcitabine to pancreatic enzymes for the treatment of pancreatic cancer. Anyone reading the paper would think that the study was well conducted and the results valid. Summarizing the trial, the National Cancer Institute (www.cancer.gov) states that, "Patients treated with standard chemotherapy survived an average of 14 months and patients treated with the Gonzalez regimen survived only an average of 4.3 months."

Although these are indeed the results reported by Chabot et al.[1], a team of physicians at Columbia who had managerial control of the trial, the study is so flawed that no conclusions about the effectiveness of the pancreatic enzyme treatment of cancer can be reached from its findings. The purpose of this paper is to review these methodological flaws, which have been discussed by Gonzalez [2] but not described in the peer-reviewed literature.

The pancreatic enzyme treatment tested in the study was based on the trophoblast model of cancer [3, 4]. The National Cancer Institute (www.cancer.gov) states that, "The Gonzalez regimen is based on the theory that pancreatic enzymes help the body get rid of toxins (harmful substances) that lead to cancer." This is not correct. The trophoblast model of cancer does not state that pancreatic enzymes rid the body of toxins. The Gonzalez regimen is based on the hypothesis that the pancreatic enzymes act on ectopic trophoblasts that have become malignant; just as they convert the trophoblast to the stable placenta during fetal development, in the adult these enzymes convert cancerous trophoblasts to a stable, non-malignant state. The adult cancer cells are not de-differentiated normal tissue cells, rather they are ectopic trophoblasts that have escaped normal regulation and taken on features of their surrounding tissue due to local signaling mechanisms.

Both the erroneous characterization of the trophoblast model of cancer and the mistaken characterization of the Chabot et al. [1] trial as valid should be corrected. This is true because of the following problems with the study:

\section{Methodological Flaws}

\subsection{Problems with the Initial Randomization Design}

A randomized, prospective, controlled trial is the gold standard for any treatment study. This was the initial design for the pancreatic enzyme trial for the first year. Although it was the best design in theory, in clinical reality, it was unmanageable. Most patients referred to the trial were interested in alternative approaches and wanted to try pancreatic enzymes. These people declined to participate once they learned that they might be randomized to chemotherapy. Conversely, prospective patients not interested in alternative approaches would not agree to participate because they would not want to 
take the risk of being randomized to pancreatic enzymes. Despite objections about these problems with randomization raised by Dr. Gonzalez and Dr. Isaacs, the Columbia team went ahead with the randomization.

During the first year of the study, a total of 262 people contacted Columbia expressing an interest in the trial, but only three were enrolled. One of these was assigned to enzyme treatment and two to chemotherapy. After that year, the Columbia physicians agreed to change to a matched cohort design, with patients allowed to choose the arm to which they would be assigned. Chabot et al. [1] mention that only three patients were enrolled in the first 14 months of the study, but they do not mention that this was 3 out of 262 people.

This number (3 out of 262) points to extreme problems enrolling patients in the trial who were suitable for and interested in pancreatic enzyme treatment, and who were willing to be randomized.

\subsection{Lack of a Lead-In Period}

Drs. Gonzalez and Isaacs argued repeatedly for the inclusion of a lead-in period for patients assigned to pancreatic enzyme treatment. This was necessary to determine whether the patients could follow the rigorous protocol, had the necessary support at home, and could eat and swallow the large number of capsules required. The Columbia physicians did not allow inclusion of a lead-in period, which resulted in recruitment of unsuitable patients into the pancreatic enzyme arm. Out of 39 patients assigned to enzyme treatment, 11 never started treatment or dropped out within the first week, yet these patients were included in the intent-to-treat analysis by the Columbia team. This problem is not mentioned by Chabot et al. [1].

\subsection{Dr. Gonzalez and Dr. Isaacs Were Not Allowed to Participate in Patient Screening}

Despite their requests to do so, Drs. Gonzalez and Isaacs were not allowed to participate in patient screening. This resulted in unsuitable patients being referred to the pancreatic enzyme arm, despite the fact that they violated written exclusion criteria agreed upon before the start of the study. This problem is not mentioned by Chabot et al. [1] the ostensible purpose for the Columbia team controlling screening was to eliminate bias; however this only eliminated any possible bias by Drs. Gonzalez and Isaacs. Any bias on the part of the Columbia team went unmonitored and unchecked.

\subsection{The Intent to Treat Analysis}

The Columbia team insisted on publishing an intent-to-treat analysis. However, this included the 11 unsuitable and wholly non-compliant patients described above. This is not a fair trial of the pancreatic enzyme protocol. Drs. Gonzalez and Isaacs do not claim that patients will benefit from their treatment protocol if they do not adhere to it. This is equivalent to including patients who take one dose of chemotherapy.

\subsection{Patient Compliance with the Pancreatic Enzyme Protocol After the First Week}

According to Gonzalez [2], out of 39 patients entered into the pancreatic enzyme arm of the trial, 30 followed the protocol either not at all, incompletely, or for very limited periods of time. The reasons for non-compliance included physical disability, psychiatric instability, lack of social support, poor motivation and pressure from family physicians and oncologists not to participate. None of this is mentioned by Chabot et al. [1]. Only one patient adhered fully to the pancreatic enzyme protocol.

\subsection{Lack of Informed Consent}

The Columbia team referred three patients for pancreatic enzyme treatment for whom there was no evidence that signed, informed consent had been obtained. No evidence of informed consent was ever provided despite extensive correspondence from Dr. Gonzalez to the Columbia team on that problem. Chabot et al. [1] only mentioned one patient being excluded because a consent form was misplaced.

\subsection{Patients Enrolled Who Met Study Exclusion Criteria}

Of 39 patients assigned to the pancreatic enzyme arm of the study, 16 met one or more exclusion criteria at the time of entry. These criteria included: patients entered more than 8 weeks after biopsy confirmation of disease; patients who could not eat adequately at the time of entry; patients with psychiatric illness; patients lacking family support; and patients with no evidence of signed, informed consent. None of these exclusion criteria violations are mentioned in Chabot et al. [1]. 


\subsection{Problems with Authorship}

Although Drs. Gonzalez and Isaacs provided the enzymatic treatment in the study, they were not offered authorship, did not read the Chabot et al. [1] paper before it was submitted to the Journal of Clinical Oncology, and only found out that it was to be published when they saw it online. This is highly unusual and is symptomatic of how the entire trial was conducted. There is no explanation in Chabot et al. [1] as to why Gonzalez and Isaacs were not co-authors.

\subsection{Bias In Favor of the Chemotherapy Arm of the Trial}

Drs. Gonzalez and Isaacs were excluded from patient screening, publication, and numerous aspects of the conduct of the trial, ostensibly to avoid bias. However, this left all control in the hands of the Columbia team that developed the chemotherapy protocol used in the trial [5]. These physicians were chemotherapists, and had no prior interest in or experience with alternative medicine. Thus, the trial was biased in favor of the chemotherapy arm.

\subsection{Stage of Disease Not Balanced Across the Two Arms of the Study}

According to Chabot et al. [1] the chemotherapy arm included $60.9 \%$ stage IV patients, while the enzyme arm included $68.7 \%$ stage IV patients, which makes the trial seem balanced. There was no significant difference between the two arms in this regard. However, there were unexplained irregularities in staging during the trial, according to Gonzalez [2]. In December, 2004, at which time the trial had been running for 5 years, Dr. Chabot provided Drs. Gonzalez and Isaacs with a report in which only $38 \%$ of the chemotherapy patients had Stage IV disease, while, according to Dr. Gonzalez [2], $76 \%$ of the 39 enzyme patients had Stage IV disease. Drs. Gonzalez and Isaacs had no access to the raw staging information on the chemotherapy patients.

By March, 2005, Dr. Chabot had disqualified 2 Stage II-III chemotherapy patients without explanation and adjusted the staging to 50\% Stage IV. From April till October, 2005, Dr. Chabot entered one patient into the enzyme arm and 11 into the chemotherapy arm, 9 of whom were Stage IV. In April, 2006 Dr. Chabot reported to Drs. Gonzalez and Isaacs that 65.5\% of patients in the chemotherapy arm were Stage IV, while 59\% of the nutrition group were Stage IV. Without explanation, Dr. Chabot had disqualified two Stage II-III enzyme patients and classified 5 as Stage IIIII who were actually Stage IV by clinical records. All these adjustments were made long after the last patient was entered and long after their entry staging was determined. By study design, the staging was supposed to be fixed at entry, and not be subject to modification. None of these problems are mentioned by Chabot et al. [1].

\subsection{Seven Patients Dropped From the Pancreatic Enzyme Arm of the Study Without Explanation}

Chabot et al. [1] report that there were 32 patients in the enzyme arm, but 39 were enrolled and seen by Drs. Gonzalez and Isaacs. No explanation was ever provided for why these seven patients were excluded from the intent-to-treat analysis.

\subsection{Survival Times Inconsistent With Prior Literature on Both Enzyme Treatment and Chemotherapy}

As reported by Chabot et al. [1], the mean survival time in the enzyme group was 4.3 months while in the chemotherapy group it was 14 months. Chabot et al. do not comment on the fact that the mean survival time in the Gonzalez and Isaacs [6] pilot study of enzyme treatment was 18 months. In a gemcitabine chemotherapy trial from the same time period, the average survival time was 5.6 months [7]. Chabot et al. [1] do not comment on the fact that these survival times were reversed in their study. Nor do Chabot et al. [1] mention that the two longest-surviving patients in the study were pancreatic enzyme patients, one of whom who had survived 173 weeks from the time of biopsy and 167.3 weeks from the time of starting enzyme treatment.

The introduction to Gonzalez [2] is written by a woman, who was diagnosed with a 3.2 centimeter pancreatic cancer tumor in December, 2000. The biopsy was done by Kaiser and confirmed at the Mayo Clinic. She was alive 13.5 years later. These outcomes are common in Drs. Gonzalez and Isaacs' practices. Rather than providing evidence that the enzyme treatment of pancreatic cancer is ineffective, Chabot et al [1]. Provides evidence that the NCI trial was flawed, and did not test the Gonzalez protocol. 


\subsection{Logistical Problems with the Study}

Besides the switch from randomization to a matched cohort design, the study is flawed because of a series of organizational and logistical problems documented by Gonzalez [2]. For example, due to delays in disbursing funds, which were entirely controlled by Columbia, Drs. Gonzalez and Isaacs had to pay for enzymes out of their own pockets on many occasions. At one point they had an accounts receivable with Columbia of over $\$ 20,000.00$ due to these delays. The study was on hold to new patient enrollment for multiple prolonged periods: during one three-year period, it was down more than it was up and running. Numerous chronic problems in communication with Drs. Gonzalez and Isaacs are documented by Gonzalez [2], along with various other organizational problems. None of these invalidate the study, but they demonstrate that the study was flawed at many different levels, and in many different ways.

\subsection{Fine-Tuning of Treatment Allowed in the Chemotherapy Arm But Not the Enzyme Arm}

During the trial, the Columbia physicians were allowed to modify the protocol if the patient was not responding adequately. However, if patients in the enzyme arm were doing poorly, no modification of their regimen was allowed and they were dropped from treatment but retained in the intent-to-treat analysis. In their clinical practice, Drs. Gonzalez and Isaacs modify their protocol on an as-needed basis; therefore the trial did not test the effectiveness of their actual practice. This double standard is not mentioned by Chabot et al. [1].

\section{Conclusion}

In conclusion, there are numerous methodological flaws in the Chabot et al. [1] study. These occur at a variety of levels from study design to study execution and reporting. A number of the flaws are so severe that they render the results invalid. No conclusions about the treatment of cancer with pancreatic enzymes can be reached from the study, which should therefore be disregarded. A second trial should be undertaken in which these flaws are corrected.

\section{REFERENCES}

[1] Chabot J.A., Tsai W.Y., Fine R.L., et al. Pancreatic proteolytic enzyme therapy compared with gemcitabine-based chemotherapy for the treatment of pancreatic cancer. J Clin Oncol. 28, 2058 (2010).

[2] N.J. Gonzalez,What Went Wrong. The Truth behind the Clinical Trial of the Enzyme Treatment of Cancer. New York: New Spring Press, 2012.

[3] N.J. Gonzalez andL.L. Isaacs,The Trophoblast and the Origins of Cancer. One Solution to the Medical Enigma of our Time. New York: New Spring Press, 2009.

[4] Ross C.A., The trophoblast model of cancer. Nutr. Cancer. 67, 61 (2015). Surg Oncol Clin N Am. 2004; 13:711-735.

[5] Fogelman D.R., Chen J., Chabot J.A., et al., The evolution of adjuvant and neoadjuvant chemotherapy and radiation for advanced pancreatic cancer: from 5-fluorouracil to GTX. Surg. Oncol. Clin. N. Am.13, 711(2004).

[6] Gonzalez N.J., Isaacs L.L., Evaluation of pancreatic proteolytic enzyme treatment of adenocarcinoma of the pancreas with nutrition and detoxification support. Nutr. Cancer. 33, 117 (1999).

[7] Burris H.A., Moore M.J., Andersen J., et al., Improvements in survival and clinical benefit with gemcitabine as first-line therapy for patients with advanced pancreas cancer: a randomized trial. Eur. J. Cancer. 33, 518 (1997). 